\title{
A Block Diagram of Electromagnetoelastic Actuator Nanodisplacement for Communications Systems
}

\author{
Sergey Mikhailovich Afonin \\ National Research University of Electronic Technology (MIET), Moscow, Russia; \\ eduems@mail.ru
}

\begin{abstract}
The parametric block diagram of the electromagnetoelastic actuator nanodisplacement or the piezoactuator is determined in contrast the electrical equivalent circuit types Cady or Mason for the calculation of the piezoelectric transmitter and receiver, the vibration piezomotor with the mechanical parameters in form the velosity and the pressure. The method of mathematical physics is used. The parametric block diagram of electromagnetoelastic actuator is obtained with the mechanical parameters the displacement and the force. The transfer functions of the electroelastic actuator are determined. The the generalized parametric block diagram, the generalized matrix equation for the electromagnetoelastic actuator nanodisplacement are obtained. The deformations of the electroelastic actuator for the nanotechnology are described by the matrix equation. Block diagram and structural-parametric model of electromagnetoelastic actuator nanodisplacement for nanodisplacement of the communications systems are obtained, its transfer functions are bult. Effects of geometric and physical parameters of electromagnetoelastic actuators and external load on its dynamic characteristics are determined. For calculations the communications systems with the piezoactuator for nanodisplacement the parametric block diagram and the transfer functions of the piezoactuator are obtained.
\end{abstract}

Keywords: Electromagnetoelastic actuator; Parametric block diagram; Matrix transfer function; Piezoactuator.

\section{Introduction}

The parametric block diagram of electromagnetoelastic actuator for nanodisplacement on the piezoelectric, piezomagnetic, electrostriction, magnetostriction effects, for example, the piezoactuator is determined in contrast electrical equivalent circuit types Cady or Mason for the calculation of the piezotransmitter and piezoreceiver, the vibration piezomotor with the mechanical parameters in form the velosity and the pressure [1 - 11]. The block diagram of electromagnetoelastic actuator is obtained with the mechanical parameters the displacement and the force [7 - 9]. The electromagnetoelastic actuator is used for precise alignment in the nanotechnology, the adaptive optics, the communications systems. The piezoactuator of nanometric movements operates based on the inverse piezoeffect, in which the motion is achieved due to deformation of the piezoactuator when an external electric voltage is applied to it [1 - 19].

Piezoactuator - piezomechanical device intended for actuation of mechanisms, systems or management based on the piezoelectric effect, converts the electrical signals into the mechanical movement or the 
S.M. Afonin; A Block Diagram of Electromagnetoelastic Actuator Nanodisplacement for Communications Systems, Transactions on Networks and Communications, Volume 6 No. 3, June (2018); pp: 1-9

force [13 - 15]. The piezoactuators for the drives of nano- and micrometric movements provide the movement range from several nanometers to tens of microns, the sensitivity of up to $10 \mathrm{~nm} / \mathrm{V}$, the loading capacity of up to $1000 \mathrm{~N}$, and the transmission band of up to $100 \mathrm{~Hz}$. The investigation of the static and dynamic characteristics of the piezoactuator is necessary for the calculation mechatronics systems of the nano- and micrometric movements for the communications systems. The piezoactuators provide high stress and speed of operation and return to the initial state when switched off; they have very low displacements. The piezoactuators are used in the majority of nanomanipulators for scanning tunneling microscopes, scanning force microscopes, and atomic force microscopes. The nanorobotic manipulators of nano- and microdisplacements with the piezoactuators are the key component in nanorobotic systems. The main requirement for nanomanipulators is to guarantee the positioning accurate to nanometers.

By solving the wave equation with allowance for the corresponding equation of the electromagnetoelasticity, the boundary conditions on loaded working surfaces of the electromagnetoelastic actuators, and the strains along the coordinate axes, it is possible to construct the structural parametric model of the actuator $[8,9,18]$. The transfer functions and the parametric block diagrams of the electromagnetoelastic actuators are obtained from the set of equations describing the corresponding structural parametric model of the actuator for the communications systems. The method of mathematical physics is applied for the solution of the wave equation of the electromagnetoelastic actuator for the communications systems with using the Laplace transform for the construction the parametric block diagram of the electromagnetoelastic actuator. As the result of the joint solution of the wave equation of the actuator with the Laplace transform, the equation of the electromagnetoelasticity, the boundary conditions on the two loaded working surfaces of the actuator, we obtain the corresponding structural-parametric model and the parametric block diagram of the electromagnetoelastic actuator.

\section{Block Diagram of Electromagnetoelastic Actuator}

The parametric block diagram and the matrix transfer functions of the electromagnetoelastic actuator for the nanotechnology are obtained from the structural-parametric model of the electromagnetoelastic actuator with the mechanical parameters the displacement and the force. The parametric block diagrams of the voltage-controlled or current-controlled piezoactuator are determined from the generalized structural-parametric model of the electromagnetoelastic actuator.

The equation of the electromagnetoelasticity of the actuator $[7,8,11]$ has the form

$$
S_{i}=s_{i j}^{E, H} T_{j}+d_{m i}^{H} E_{m}+d_{m i}^{E} H_{m}
$$

where $S_{i}$ is the relative deformation along the axis $i, E$ is the electric field strength, $H$ is the magnetic field strength, $s_{i j}^{E, H}$ is the elastic compliance for $E=$ const , $H=$ const , $T_{j}$ is the mechanical stress along the axis $j, d_{m i}^{H}$ is the piezomodule, i.e., the partial derivative of the relative deformation with respect to the electric field strength for constant magnetic field strength, i.e., for $H=$ const, $E_{m}$ is the electric field strength along the axis $m, d_{m i}^{E}$ is the magnetostriction coefficient, $H_{m}$ is the magnetic field strength along the axis $m, i=1,2, \ldots, 6, j=1,2, \ldots, 6, m=1,2,3$.

Let us consider separately effect the electric field strength and the magnetic field strength, therefore the generalized equation of the electromagnetoelasticity has the form 


$$
S_{i}=d_{m i} \Psi_{m}(t)+s_{i j}^{\Psi} T_{j}(x, t)
$$

where $S_{i}=\partial \xi(x, t) / \partial x$ is the relative displacement along axis $i$ of the cross section of the piezoactuator, $\Psi_{m}=\left\{E_{m}, D_{m}, H_{m}\right.$ is the control parameter $E_{m}$ for the voltage control, $D_{m}$ for the current control, $H_{m}$ for magnetic field strength control along axis $m, T_{j}$ is the mechanical stress along axis $j, d_{m i}$ is the coefficient of electromagnetoelasticity, for example, piezomodule, $s_{i j}^{\Psi}$ is the elastic compliance for the control parameter $\Psi=$ const .

The main size is determined us the working length $l=\{\delta, h, b$ for the actuator or the piezoactuator in form the thickness, the height and the width for the longitudinal, transverse and shift the piezoeffect.

For the construction the parametric block diagram of the electromagnetoelastic actuator in nanotechnology is used the wave equation for the wave propagation in a long line with damping but without distortions. With using Laplace transform is obtained the linear ordinary second-order differential equation with the parameter $s$. Correspondingly the original problem for the partial differential equation of hyperbolic type using the Laplace transform is reduced to the simpler problem $[8,14,18]$ for the linear ordinary differential equation

$$
\mathrm{d}^{2} \Xi(x, s) / \mathrm{d} x^{2}-\gamma^{2} \Xi(x, s)=0
$$

with its solution

$$
\Xi(x, s)=C e^{-x y}+B e^{x y}
$$

where $\Xi(x, s)$ is the Laplace transform of the displacement of section of the actuator, $\gamma=s / c^{\Psi}+\alpha$ is the propagation coefficient, $C^{\Psi}$ is the sound speed for $\Psi=$ const, $\alpha$ is the damping coefficient, $C$ and $B$ are constants.

From generalized electromagnetoelasticity equation (3), we obtain the system of equations describing the generalized structural-parametric model of the electromagnetoelastic actuator for the communications systems:

$$
\begin{aligned}
& \Xi_{1}(s)=\left[1 /\left(M_{1} s^{2}\right)\right] \times \\
& \times\left\{-F_{1}(s)+\left(1 / \chi_{i j}^{\Psi}\right)\left[v_{m i} \Psi_{m}(s)-[\gamma / \operatorname{sh}(l \gamma)]\left[\operatorname{ch}(l \gamma) \Xi_{1}(s)-\Xi_{2}(s)\right]\right]\right\} \\
& \Xi_{2}(s)=\left\lfloor 1 /\left(M_{2} s^{2}\right)\right\rfloor \times \\
& \times\left\{-F_{2}(s)+\left(1 / \chi_{i j}^{\Psi}\right)\left[v_{m i} \Psi_{m}(s)-[\gamma / \operatorname{sh}(l \gamma)]\left[\operatorname{ch}(l \gamma) \Xi_{2}(s)-\Xi_{1}(s)\right]\right]\right\} \\
& \text { where } \quad v_{m i}=\left\{\begin{array}{l}
d_{33}, d_{31}, d_{15} \\
g_{33}, g_{31}, g_{15} \\
d_{33}, d_{31}, d_{15}
\end{array}, \Psi_{m}=\left\{\begin{array}{l}
E_{3}, E_{1} \\
D_{3}, D_{1}, s_{i j}^{\Psi} \\
H_{3}, H_{1}
\end{array}=\left\{\begin{array}{l}
s_{33}^{E}, s_{11}^{E}, s_{55}^{E} \\
s_{33}^{D}, s_{11}^{D}, s_{55}^{D}, c^{\Psi} \\
s_{33}^{H}, s_{11}^{H}, s_{55}^{H}
\end{array}=\left\{\begin{array}{l}
c^{E} \\
c^{D} \\
c^{H}
\end{array}, \gamma=\left\{\begin{array}{l}
\gamma^{E} \\
\gamma^{D} \\
\gamma^{H}
\end{array}, l=\left\{\begin{array}{l}
\delta \\
h, \chi_{i j}^{\Psi}=s_{i j}^{\Psi} \\
b
\end{array} s_{0},\right.\right.\right.\right.\right.\right.
\end{aligned}
$$

then parameters $\Psi_{m}=\left\{E_{m}, D_{m}, H_{m}\right.$ of the control for the electromagnetoelastic actuator, $v_{m i}$ is the coefficient of the electromagnetoelasticity, for example, $d_{m i}$ is the piezomodule for the voltagecontrolled piezoactuator, $g_{m i}$ is the piezomodule for the current-controlled piezoactuator, $d_{m i}$ is the coefficient of the magnetostriction, $S_{0}$ is the cross section area and $M_{1}, M_{2}$ are the displaced mass on 
S.M. Afonin; A Block Diagram of Electromagnetoelastic Actuator Nanodisplacement for Communications Systems, Transactions on Networks and Communications, Volume 6 No. 3, June (2018); pp: 1-9

the faces of the electromagnetoelastic actuator, $\Xi_{1}(s), \Xi_{2}(s)$ and $F_{1}(s), F_{2}(s)$ are the Laplace transform of the displacements and the forces on the faces of the electromagnetoelastic actuator. Figure 1 shows the generalized parametric block diagram of the electromagnetoelastic actuator corresponding to the equation (2) of the electromagnetoelasticity and the set of equations (5).

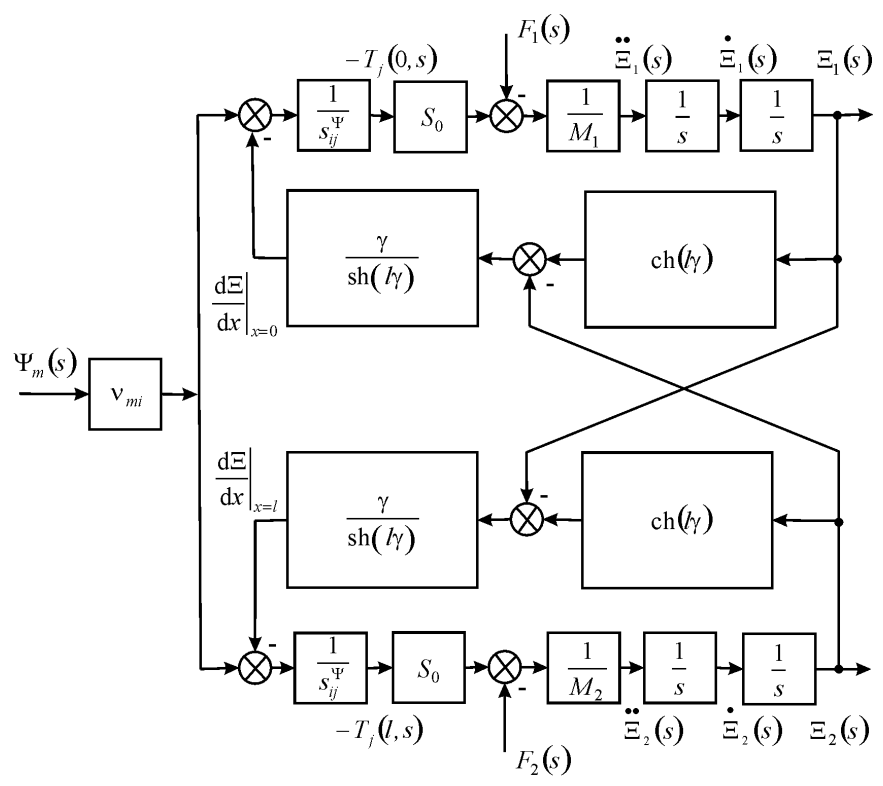

Figure 1. Generalized parametric block diagram of electromagnetoelastic actuator for nanodisplacement

The matrix state equations $[11,14]$ for the piezoelectric effect have the form

$$
\begin{aligned}
& (\mathbf{D})=(\mathbf{d})(\mathbf{T})+\left(\boldsymbol{\varepsilon}^{T}\right)(\mathbf{E}) \\
& (\mathbf{S})=\left(\mathbf{s}^{E}\right)(\mathbf{T})+(\mathbf{d})^{t}(\mathbf{E})
\end{aligned}
$$

where the equation (6) describes the direct piezoeffect, the equation (7) depicts the inverse piezoeffect, and the matrixes: (D) is the column-matrix of the electric induction along the coordinate axes, $(\mathbf{S})$ is the column-matrix of the relative deformations, $(\mathbf{T})$ is the column-matrix of the mechanical stresses, $(\mathbf{E})$ is the column-matrix of the electric field strength along the coordinate axes, $(\mathbf{d})^{t}$ is the transposed matrix of the piezoelectric modules, $\left(\mathbf{s}^{E}\right)$ is the elastic compliance matrix, $\left(\boldsymbol{\varepsilon}^{T}\right)$ is the matrix of the dielectric constants. In the equation (6), (7) are the six stress components $T_{1}, T_{2}, T_{3}, T_{4}, T_{5}, T_{6}$. The components $T_{1}-T_{3}$ are defined to extension-compression stresses, the components $T_{4}-T_{6}$ are related to shear stresses $[8,11]$.

Let us consider the transverse piezoelectric effect in the piezoactuator. The equation of the inverse transverse piezoeffect $[8,11]$

$$
S_{1}=d_{31} E_{3}(t)+s_{11}^{E} T_{1}(x, t)
$$

where $\left.S_{1}=\partial \xi(x, t)\right) / \partial x$ is the relative displacement of the cross section of the piezoactuator along axis 1 , $d_{31}$ is the piezoelectric module for the transverse piezoeffect, $s_{11}^{E}$ is the elastic compliance for $E=$ const along axis $1, T_{1}$ is the stress along axis 1. 
The solution of the linear ordinary second-order differential equation with the parameter s (3) we obtain as (4) and subject to the conditions

$$
\begin{aligned}
& \Xi(0, s)=\Xi_{1}(s) \text { for } x=0 \\
& \Xi(h, s)=\Xi_{2}(s) \text { for } x=h
\end{aligned}
$$

The constants $C$ and $B$ for the solution are determined in the following form

$$
C=\left(\Xi_{1} e^{h \gamma}-\Xi_{2}\right) /[2 \operatorname{sh}(h \gamma)], B=\left(\Xi_{2}-\Xi_{1} e^{-h \gamma}\right) /[2 \operatorname{sh}(h \gamma)]
$$

Then, the solution (4) can be written as

$$
\Xi(x, s)=\left\{\Xi_{1}(s) \operatorname{sh}[(h-x) \gamma]+\Xi_{2}(s) \operatorname{sh}(x \gamma)\right\} / \operatorname{sh}(h \gamma)
$$

The equations of forces acting on the faces of the piezoactuator has the form

$$
\begin{aligned}
& T_{1}(0, s) S_{0}=F_{1}(s)+M_{1} p^{2} \Xi_{1}(s) \text { for } x=0 \\
& T_{1}(h, s) S_{0}=-F_{2}(s)-M_{2} s^{2} \Xi_{2}(s) \text { for } x=h
\end{aligned}
$$

where $T_{1}(0, s)$ and $T_{1}(h, s)$ are determined from the equation of the inverse transverse piezoeffect.

Therefore we obtain the system of the equations for the mechanical stresses at the faces of the piezoactuator for the transverse piezoeffect in the form

$$
\begin{aligned}
& T_{1}(0, s)=\left(1 / s_{11}^{E}\right) \mathrm{d} \Xi(x, s) /\left.\mathrm{d} x\right|_{x=0}-d_{31} E_{3}(s) / s_{11}^{E} \\
& T_{1}(h, s)=\left(1 / s_{11}^{E}\right) \mathrm{d} \Xi(x, s) /\left.\mathrm{d} x\right|_{x=h}-d_{31} E_{3}(s) / s_{11}^{E}
\end{aligned}
$$

The set of equations (13) for mechanical stresses in piezoactuator yields the following set of equations describing the structural parametric model and the parametric block diagram of the voltage-controlled piezoactuator for the transverse piezoelectric effect on Figure 2

$$
\begin{aligned}
& \Xi_{1}(s)=\left[1 /\left(M_{1} s^{2}\right)\right] \times \\
& \times\left\{-F_{1}(s)+\left(1 / \chi_{11}^{E}\right)\left[d_{31} E_{3}(s)-[\gamma / \operatorname{sh}(h \gamma)]\left[\operatorname{ch}(h \gamma) \Xi_{1}(s)-\Xi_{1}(s)\right]\right\}\right. \\
& \Xi_{2}(s)=\left[1 /\left(M_{2} s^{2}\right)\right] \times \\
& \times\left\{-F_{2}(s)+\left(1 / \chi_{11}^{E}\right)\left[d_{31} E_{3}(s)-[\gamma / \operatorname{sh}(h \gamma)]\left[\operatorname{ch}(h \gamma) \Xi_{2}(s)-\Xi_{1}(s)\right]\right]\right\}
\end{aligned}
$$

where $\chi_{11}^{E}=s_{11}^{E} / S_{0}, l=h$. 


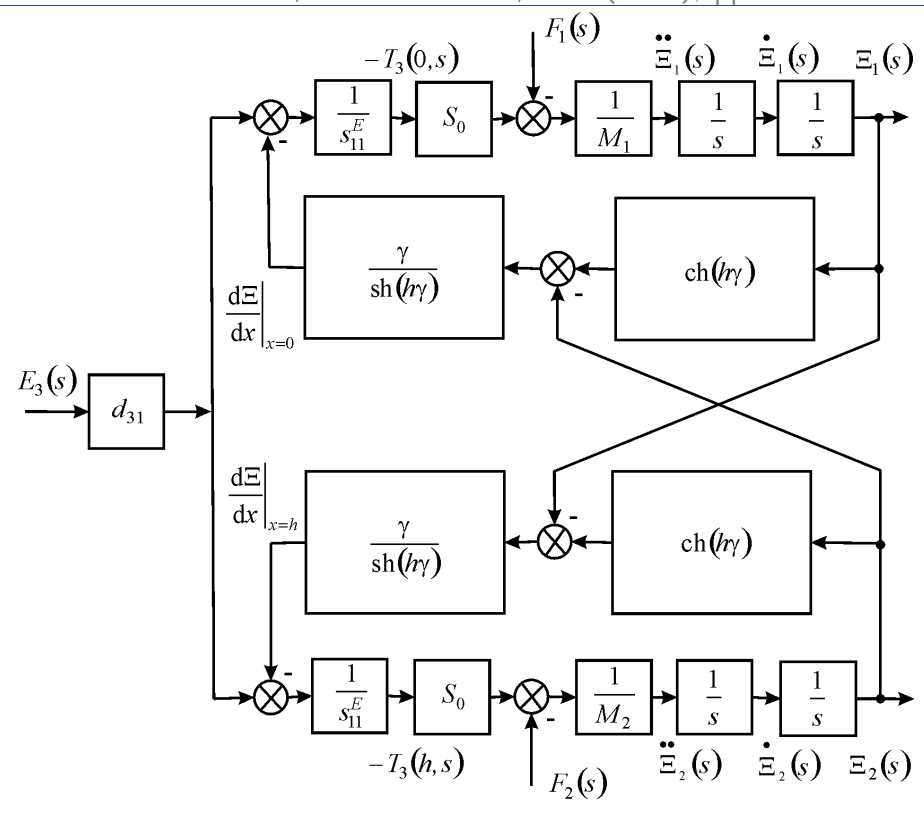

Figure 2. Parametric block diagram of voltage-controlled piezoactuator for transverse piezoeffect

The parametric block diagram of the voltage-controlled or the current-controlled piezoactuator for the transverse, longitudinal, shift piezoelectric effects are determined from the generalized structuralparametric model of the electromagnetoelastic actuator.

\section{Matrix transfer function of electromagnetoelastic actuator}

From (5), (14) matrix equation of the Laplace transforms of the displacements with the matrix transfer function of the electromagnetoelastic actuator is obtained in the form

$$
\begin{aligned}
(\Xi(s))=(W(s))(P(s)) & \\
(\Xi(s))=\left(\begin{array}{l}
\Xi_{1}(s) \\
\Xi_{2}(s)
\end{array}\right),(W(s)) & =\left(\begin{array}{lll}
W_{11}(s) & W_{12}(s) & W_{13}(s) \\
W_{21}(s) & W_{22}(s) & W_{23}(s)
\end{array}\right) \\
(P(s)) & =\left(\begin{array}{c}
\Psi_{m}(s) \\
F_{1}(s) \\
F_{2}(s)
\end{array}\right)
\end{aligned}
$$

where $(\Xi(s))$ is the column-matrix of the Laplace transforms of the displacements, $(W(s))$ is the matrix transfer function, $(P(s))$ the column-matrix of the Laplace transforms of the control parameter and the forces.

The generalized transfer functions of the electromagnetoelastic actuator are the ratio of the Laplace transform of the displacement of the face and the Laplace transform of the corresponding control parameter or the force at zero initial conditions

$$
\begin{gathered}
W_{11}(s)=\Xi_{1}(s) / \Psi_{m}(s)=v_{m i}\left[M_{2} \chi_{i j}^{\Psi} s^{2}+\gamma \operatorname{th}(l \gamma / 2)\right] / A_{i j} \\
\chi_{i j}^{\Psi}=s_{i j}^{\Psi} / S_{0}
\end{gathered}
$$




$$
\begin{gathered}
A_{i j}=M_{1} M_{2}\left(\chi_{i j}^{\Psi}\right)^{2} s^{4}+\left\{\left(M_{1}+M_{2}\right) \chi_{i j}^{\Psi} /\left[c^{\Psi} \operatorname{th}(l \gamma)\right]\right\} s^{3}+ \\
+\left[\left(M_{1}+M_{2}\right) \chi_{i j}^{\Psi} \alpha / \operatorname{th}(l \gamma)+1 /\left(c^{\Psi}\right)^{2}\right] s^{2}+2 \alpha s / c^{\Psi}+\alpha^{2} \\
W_{21}(s)=\Xi_{2}(s) / \Psi_{m}(s)=v_{i j}\left[M_{1} \chi_{i j}^{\Psi} s^{2}+\gamma \operatorname{th}(l \gamma / 2)\right] / A_{i j} \\
W_{12}(s)=\Xi_{1}(s) / F_{1}(s)=-\chi_{i j}^{\Psi}\left[M_{2} \chi_{i j}^{\Psi} s^{2}+\gamma / \operatorname{th}(l \gamma)\right] / A_{i j} \\
W_{13}(s)=\Xi_{1}(s) / F_{2}(s)= \\
=W_{22}(s)=\Xi_{2}(s) / F_{1}(s)=\left[\chi_{i j}^{\Psi} \gamma / \operatorname{sh}(l \gamma)\right] / A_{i j} \\
W_{23}(s)=\Xi_{2}(s) / F_{2}(s)=-\chi_{i j}^{\Psi}\left[M_{1} \chi_{i j}^{\Psi} s^{2}+\gamma / \operatorname{th}(l \gamma)\right] / A_{i j}
\end{gathered}
$$

The static displacements of the faces for the voltage-controlled the piezoactuator for the shift piezoeffect are obtained from (15) at $m<M_{1}$ and $m<<M_{2}$ in the form

$$
\begin{aligned}
& \xi_{1}(\infty)=\left(M_{2} U_{0} d_{15} b / \delta\right) /\left(M_{1}+M_{2}\right) \\
& \xi_{2}(\infty)=\left(M_{1} U_{0} d_{15} b / \delta\right) /\left(M_{1}+M_{2}\right)
\end{aligned}
$$

For the voltage-controlled the piezoactuator from PZT under the shift piezoeffect at $m<<M_{1}$ and $m<<M_{2}, d_{15}=4 \cdot 10^{-10} \mathrm{~m} / \mathrm{V}, b / \delta=10, U=50 \mathrm{~V}, M_{1}=1 \mathrm{~kg}$ and $M_{2}=4 \mathrm{~kg}$ the static displacements of the faces the piezoactuator are determined $\xi_{1}(\infty)=160 \mathrm{~nm}, \xi_{2}(\infty)=40 \mathrm{~nm}, \xi_{1}(\infty)+\xi_{2}(\infty)=200 \mathrm{~nm}$.

The transfer function of the voltage-controlled transverse piezoactuator is obtained from (14) for elasticinertial load at $M_{1} \rightarrow \infty, m<<M_{2}$ and the approximation the hyperbolic cotangent by two terms of the power series in the form

$$
\begin{gathered}
W(s)=\Xi_{2}(s) / U(p)=k_{t} /\left(T_{t}^{2} s^{2}+2 T_{t} \xi_{t} s+1\right) \\
k_{t}=\left(d_{31} h / \delta\right) /\left(1+C_{e} / C_{11}^{E}\right), \quad T_{t}=\sqrt{M_{2} /\left(C_{e}+C_{11}^{E}\right)}, \quad \xi_{t}=\alpha h^{2} C_{11}^{E} /\left(3 c^{E} \sqrt{M_{2}\left(C_{e}+C_{11}^{E}\right)}\right)
\end{gathered}
$$

where $U(p)$ is the Laplace transform of the voltage, $k_{t}$ is the value of the coefficient of the displacement for the voltage-controlled piezoactuator, $T_{t}$ is the time constant and $\xi_{t}$ is the damping coefficient of the piezoactuator.

For the voltage-controlled transverse piezoactuator at $M_{1} \rightarrow \infty, m<<M_{2}$ and $d_{31}=2.5 \cdot 10^{-10} \mathrm{~m} / \mathrm{V}$, $h / \delta=20, M_{2}=1 \mathrm{~kg}, C_{11}^{E}=2 \cdot 10^{7} \mathrm{~N} / \mathrm{m}, C_{e}=0.5 \cdot 10^{7} \mathrm{H} / \mathrm{m}$ we obtain the value of the coefficient of the displacement and the time constant of the actuator $k_{t}=4 \mathrm{~nm} / \mathrm{V}, T_{t}=0.2 \cdot 10^{-3} \mathrm{c}$.

The matrix transfer function of the actuator is determined for control systems with the electromagnetoelastic actuator in the communications systems.

The generalized parametric block diagram and generalized structural-parametric model of the electromagnetoelastic actuator are obtained. From generalized structural-parametric model of the actuator after algebraic transformations the transfer functions of the actuator are determined. 
S.M. Afonin; A Block Diagram of Electromagnetoelastic Actuator Nanodisplacement for Communications Systems, Transactions on Networks and Communications, Volume 6 No. 3, June (2018); pp: 1-9

The parametric block diagram, the structural-parametric models of the piezoactuator for the transverse, longitudinal, shift piezoelectric effects are determined from the generalized structural-parametric model of the electromagnetoelastic actuator for the communications systems.

\section{Conclusion}

The generalized parametric block diagram and the generalized structural-parametric model of the electromagnetoelastic actuator are constructed with the mechanical parameters the displacement and the force.

The parametric block diagrams of the piezoactuator for the transverse, longitudinal, shift piezoelectric effects are determined. The matrix transfer function of the electromagnetoelastic actuator is obtained for the communications systems.

\section{REFERENCES}

[1] Schultz, J., Ueda, J., Asada, H., Cellular actuators. Oxford: Butterworth-Heinemann Publisher, 2017. 382 p.

[2] Afonin, S.M., Absolute stability conditions for a system controlling the deformation of an elecromagnetoelastic transduser. Doklady mathematics, 2006. 74(3): p. 943-948, doi:10.1134/S1064562406060391.

[3] Zhou, S., Yao, Z., Design and optimization of a modal-independent linear ultrasonic motor. IEEE transaction on ultrasonics, ferroelectrics, and frequency control, 2014. 61(3): p. 535-546, doi:10.1109/TUFFC.2014.2937.

[4] Przybylski, J., Static and dynamic analysis of a flextensional transducer with an axial piezoelectric actuation. Engineering structures, 2015. 84: p. 140-151, doi:10.1016/j.engstruct.2014.11.025.

[5] Ueda, J., Secord, T., Asada, H.H., Large effective-strain piezoelectric actuators using nested cellular architecture with exponential strain amplification mechanisms. IEEE/ASME transactions on mechatronics, 2010. 15(5): p. 770-782, doi:10.1109/TMECH.2009.2034973.

[6] Karpelson, M., Wei, G.-Y., Wood, R.J., Driving high voltage piezoelectric actuators in microrobotic applications. Sensors and actuators A: Physical, 2012. 176: p. 78-89, doi:10.1016/j.sna.2011.11.035.

[7] Afonin, S.M., Block diagrams of a multilayer piezoelectric motor for nano-and microdisplacements based on the transverse piezoeffect. Journal of computer and systems sciences international, 2015. 54(3): p. 424-439, doi:10.1134/S1064230715020021.

[8] Afonin, S.M., Structural parametric model of a piezoelectric nanodisplacement transduser. Doklady physics, 2008. 53(3) p. 137-143, doi:10.1134/S1028335808030063.

[9] Afonin, S.M., Solution of the wave equation for the control of an elecromagnetoelastic transduser. Doklady mathematics, 2006. 73(2), p. 307-313, doi:10.1134/S1064562406020402.

[10] Cady W.G., Piezoelectricity: An introduction to the theory and applications of electromechancial phenomena in crystals. New York, London: McGraw-Hill Book Company, 1946. 806 p. 
[11] Physical acoustics: Principles and methods. Vol.1. Part A. Methods and devices. Mason, W., Editor, New York: Academic Press, 1964. 515 p.

[12] Zwillinger, D., Handbook of differential equations. Boston: Academic Press, 1989. 673 p.

[13] Afonin, S.M., Structural-parametric model and transfer functions of electroelastic actuator for nano-and microdisplacement. Chapter 9 in Piezoelectrics and nanomaterials: Fundamentals, developments and applications. Parinov, I.A., Editor, New York: Nova Science, 2015. p. 225-242.

[14] Afonin, S.M., A structural-parametric model of electroelastic actuator for nano- and microdisplacement of mechatronic system. Chapter 8 in Advances in nanotechnology. Volume 19. Bartul, Z., Trenor, J., Editors, New York: Nova Science, 2017. p. 259-284.

[15] Afonin, S.M., Nano- and micro-scale piezomotors. Russian engineering research, 2012. 32(7-8): p. 519522, doi:10.3103/S1068798X12060032.

[16] Afonin, S.M., Elastic compliances and mechanical and adjusting characteristics of composite piezoelectric transducers. Mechanics of solids, 2007. 42(1): p. 43-49, doi:10.3103/\$0025654407010062.

[17] Afonin, S.M., Stability of strain control systems of nano-and microdisplacement piezotransducers. Mechanics of solids, 2014. 49(2): p. 196-207, doi:10.3103/S0025654414020095.

[18] Afonin, S.M., Structural-parametric model electromagnetoelastic actuator nanodisplacement for mechatronics. International journal of physics, 2017. 5(1): p. 9-15, doi: 10.12691/ijp-5-1-2.

[19] Springer Handbook of Nanotechnology. Bhushan, B., Editor, Springer, Berlin, New York, 2004. 1222 p. 